\title{
Die Erscheinungen bei der elektrolytischen Zersetzung von Chlorwasserstoffsäure.
}

\author{
Von \\ F. A. Gooch und F. L. Gates. ${ }^{1}$ \\ Mit 2 Figuren im Text.
}

Nach der herrschenden Theorie der Elektrolyse werden alle Ionen der Lösung, von welcher Natur sie auch seien, von den elektrischen Kräften angegriffen und alle führen den Strom, indem sie sich durch die Flüssigkeit bewegen. Wenn mehr als eine Art von Ionen in der Lösung vorhanden sind, so wird diejenige Art, die die niedrigste Zersetzungsspannung hat, zuerst an der Elektrode abgeschieden.

Bei der Zersetzung von Chlorwasserstoffsäure gehen die Wasserstoffionen der Säure zur Kathode und verwandeln sich dort in neutralen Wasserstoff. Die Chlorionen gehen an die Anode, und wenn die Lösung ziemlich konzentriert ist, so werden sie dort entladen und in neutrales Chlor übergeführt. Unter diesen Verhältnissen haben die Hydroxylionen des Lösungsmittels Wasser eine höhere Zersetzungsspannung als die Chlorionen und beteiligen sich demnach an der Überführung nur in unbedeutendem Marse. Wenn die Konzentration bis zu dem Punkte fällt, wo die Diffusion der Säure in Lösung nicht mehr ausreicht, um die Chlorionen zu ersetzen, die aus der der Anode anliegenden Flüssigkeitsschicht entfernt sind, so können die Hydroxylionen des Wassers am Transport des Stromes aus der Lösung in die Elektrode teilnehmen und die Polarisation steigt, bis sie in aufserordentlich verdünnter Lösung die Zersetzungsspannung des Wassers erreicht. Aus starken Lösungen von Chlorwasserstoffsäure entweicht an der Kathode Wasserstoff und an der Anode Chlor, während mit abnehmender Konzentration Sauerstoff aus Hydroxyl an die Stelle von Chlor tritt. Le Blanc hat experimentell gezeigt, dafs die folgenden Werte der Zersetzungsspannung für verschiedene Konzentrationen der Salzsäure gelten:

${ }^{1}$ Aus dem Amer. Journ. Sci. (Sill.) ins Deutsche übertragen von I. KoppeLBerlin. 
Zersetzungsspannung

2-norm. $\mathrm{HCl}$

$1 / 2$-norm. $\mathrm{HCl}$

$1 / 6$ - norm. $\mathrm{HCl}$

$1 / 16$ - norm. $\mathrm{HCl}$

$1 / 33^{-n o r m} . \mathrm{HCl}$
1.26 Volt

1.34 ,

1.41,

1.62 "

1.69,

Nach dieser Theorie wird der Transport des Stromes von der Lösung zur Elektrode bei der höchsten Verdünnung primär durch die Ionen des Wassers besorgt, während im Innern der Lösung der Strom fast vollständig durch die Ionen der Säure fortgeführt wird.

Eine neuere Reihe von Arbeiten von Doumer ${ }^{1}$ enthält die Berichte von Versuchen über die Elektrolyse von Chlorwasserstoffsäure und verschiedene daraus abgeleitete Schlüsse über die Rolle des Wassers beim Transport des elektrischen Stromes und über die Wanderungsgeschwindigkeit des Chlor- und Wasserstoffions. Bei den zuerst mitgeteilten Versuchen ${ }^{2}$ wurde eine Elektrode von Platindraht, $0.5 \mathrm{~mm}$ im Durchmesser und $6 \mathrm{~cm}$ lang, benutzt; bei diesem Versuch ergab sich, dals die freigemachten Volumina Sauerstoff an der Anode bei Strömen zwischen 0.120 und 0.134 Amp., die ungefähr 1 Stunde hindurchgingen, $z \mathfrak{u}$ den gleichzeitig an der Kathode freigemachten Wasserstoffvolumina ein Verhältnis zeigten, das mit der Konzentration der Lösung wechselte. Nimmt man das entwickelte Wasserstoffvolumen als Einheit, so sind die Verhältnisse der Volumina von Sauerstoff und Wasserstoff für die verschiedenen Konzentrationen folgende:

$\begin{array}{llccccl}\text { Konzentration } \% & 14.5 & 8.7 & 5.8 & 2.9 & 1.45(?) & 0.72 \\ \text { Verhältnis von O:H } & 0.034 & 0.068 & 0.082 & 0.120 & 0.166 & 0.212\end{array}$

Ähnliche Resultate wurden erhalten bei einem anderen Versuch, bei dem eine Silberanode, deren Dimensionen nicht angegeben sind, zur Aufnahme des Chlors diente; aber die Entwickelung von Sauerstoff erwies sich als relativ grölser, indem das Verhältnis für eine Konzentration von 0.72 Teilen Chlorwasserstoffsäure in 1000 Teilen der Lösung 0.253 war, während dies Verhältnis 0.212 betrug bei Anwendung einer Platinanode. Aus der Tatsache, dals die Entwickelung von Sauerstoff nicht aufhörte, sondern ziemlich stark unter solchen Bedingungen vergrölsert wurde, zog Doumer den

1 Compt. rend. 146, 687. 897.

2 Compt. rend. 146, 329-331. 
Schlufs, dafs das Auftreten des Sauerstoffs bei der Elektrolyse von Chlorwasserstoffsäure nicht auf die sekundäre Wirkung des Chlors auf das Wasser der Lösung zurückgefübrt werden könnte, und dafs hier eine direkte elektrolytische Zersetzung des Wassers wie auch der Säure stattfinde.

In einem folgenden Artikel ${ }^{2}$ wird über eine ähnliche Versuchs. reihe mit Silberanode berichtet, wo Ablesungen des entwickelten Gases in 20 aufeinander folgenden Zeiträumen von je 5 Minuten vorgenommen wurden. Es wurde festgestellt, dafs, wenn eine Silberanode für die Elektrolyse von Chlorwasserstoffsäure benutzt wird, sich braunes Silberoxyd bildet, bis der Niederschlag ron Oxyd und Chlorid auf der Anode eine Dicke erreicht, welche er nicht überschreiten zu können scheint und dals dann die Entwickelung des Sauerstoffs konstant wird, während das bei der Elektrolyse entwickelte Chlor an der Anode als Silberchlorid haften bleibt. Die Flüssigkeit enthält keine Spur von freiem Chlor oder ron Oxychloriden, wenn schwache Ströme gebraucht werden. Während der ersten 20 Minuten der vorläufigen Elektrolyse wurden bei dem fraglichen Versuche die entwickelten Gasvolumina nicht beobachtet. Für die ersten fünf folgenden Perioden waren die volumetrischen Verhältnisse von Sauerstoff zu Wasserstotf $0.247,0.272,0.285,0.319$. Später, in den übrigen 15 Intervallen blieben die Sauerstoff-Wasserstoffverhältnisse fast konstant im Mittel: 0.332. Praktisch derselbe Mittelwert (0.331) wird für die Beobachtungen angegeben, die unter verschiedenen Stromverhältnissen und Konzentrationen der Salzsäure gemacht wurden, nämlich bei einem Strom von $0.006 \mathrm{Amp}$. und $0.023 \mathrm{Amp}$. in einer Lösung von 1.25 auf 1000 ; für $0.021 \mathrm{Amp}$. und $0.250 \mathrm{Amp}$. in einer Lösung von $5.3 \mathrm{zu} 1000$; bei $0.020 \mathrm{Amp}$. und $0.100 \mathrm{Amp}$. in einer Lösung von $10.7 \mathrm{zu} 1000$ und bei $0.053 \mathrm{Amp}$. in einer Lösung von 55.8 auf 1000. Demnach wurde der Schlufs gezogen, dafs das Verbältnis der Volumina von Sauerstoff an der Anode zum Volumen des Wasserstoffs an der Kathode bei der Elektrolyse von Chlorwasserstoffsäure konstant ist, und unabhängig von der Intensität des Stromes und der Konzentration der Lösung, und da ein folgendes Experiment mit einer Quecksilberkathode ein genau übereinstimmendes Verhältnis lieferte, so wurde weiter geschlossen, dafs das Verhältnis auch vielleicht von der Natur der Anode unabhängig wäre.

1 Compt. rend. 146, 687-690. 
Das Verhältnis des Wassersoffvolumens, das dem freigemachten Sauerstoff äquivalent ist, zum Gesamtvolumen des Wasserstoffs - als Einheit - nennt Doumer den „Faktor der Ionisation des Wassers" und dieser Faktor 0.662 oder ungefähr $2 / 3$ drückt die Ansicht aus, dafs von dem entwickelten Wasserstoff an der Kathode ungefähr $2 / 3$ aus dem Wasser stammen und $1 / 3$ aus Chlorwasserstoffsäure, und dafs für jedes Molekül Chlorwasserstoffsäure, das elektrolysiert wird, auch 1 Molekül Wasser elektrolysiert werden mufs, wenn Wasser in $2 \mathrm{H}^{\circ}$ and $\mathrm{O}^{\prime}$ ionisiert wird, oder zwei Molekel, wenn Wasser in $\mathrm{H}^{\cdot}$ and $\mathrm{OH}^{\prime}$ zerfällt.

Eine spätere Mitteilung ${ }^{1}$ beschäftigt sich mit der Geschwindigkeit der Bewegung der Chlor- und Wasserstoffionen. Unter der Annahme, dafs $2 / 3$ des Stromes von den Ionen des Wassers transportiert werden und $1 / 3$ von den Ionen der Salzsäure, berechnet Doumer, dals der Verlust an Säure an beiden Elektroden derselbe sein sollte. Dieses fand sich auch bei drei Versuchen mit einer Silberanode in verdünnten Lösungen bei schwachen Strömen. Doumer fufst daher seine Ergebnisse folgendermafsen zusammen: 1. Die Ionisation des Wassers beeinflufst in aktiver Form die Elektrolyse der Salzsäurelösungen und 2. die Geschwindigkeit der Überführung der Ionen $\mathrm{H}^{\cdot}$ und $\mathrm{Cl}^{\prime}$ ist praktisch dieselbe.

Die neueren und sehr sorgfältigen Überführungsversuche von NoYes und SAMMET $^{2}$ führen zu genau denselben Schlüssen über die Ionengeschwindigkeit, vorausgesetzt, dafs man annimmt, dafs $2 / 3$ des Stromes in der Lösung durch die Ionen des Wassers transportiert werden. Bei diesen Versuchen, wo Salzsäurelösungen von bekanntem Gehalt zwischen einer Platinkathode und einer Anode aus einer Silberscheibe von ungefähr $3.5 \mathrm{~cm}$ Durchmesser mit einem Strom von nicht über 4.5 Milliampere auf das Quadratzentimeter elektrolysiert wurden, entwickelte sich kein Chlor, wohl aber etwas Sauerstoff und in jedem Falle bildete Silberchlorid eine Wolke um die Anode. Die Überführungszahl, berechnet aus der Gesamtmenge des Silbers im Voltameter und der Änderung der Stärke der Säure an der Kathode stimmte sehr genau mit der Zahl überein, die basiert war auf dem Vergleich der Voltameteranzeige mit der Änderung des Chlorgehaltes des Anodenmateriales, welche gefunden wurde durch Bestimmung des festhaftenden Silberchlorids zu-

1 Compt. rend. 146, 894-896.

${ }^{2}$ Journ. Amer. Chem. Soc. 24, 949. 
sammen mit dem Niederschlag, den Silbernitrat in der Anodenflüssigkeit hervorrief. Die Titration der Anodenflüssigkeit auf acidimetrischem Wege gab jedoch aufserordentlich wenig übereinstimmende Resultate, die wahrscheinlich auf das Auftreten von etwas Sauerstoff an der Elektrode und die Bildung einer entsprechenden Menge Säure zurückzuführen sind. Die Überführungszahl für Chlor, berechnet unter der Annahme, dafs der ganze Strom von den Ionen der Chlorwasserstoffsäure transportiert wird, wechselte etwas mit Verdünnung und Temperatur; bei $29^{\circ}$ war sie im Mittel 166.6 (für $1 / 20^{-}$und $1 / 60^{-n o r m . ~ L o ̈ s u n g e n) ; ~ w e n n ~ m a n ~ a b e r ~ m i t ~}$ DOUMER annahm, dafs $1 / 3$ des hindurchgehenden Stromes für die Elektrolyse der Salzsäure verwendet wird, und wenn man $1 / 3$ der ganzen Angabe des Voltameters als Mals des allein für die Elektrolyse der Salzsäure verwendeten Stromes annimmt, so würde die Überführungszahl des Chlors 499.8 werden und würde ebenso wie Doumers direkte Prüfung der Acidität an der Anode und Kathode darauf hinweisen, dafs Wasserstoff- und Chlorionen dieselbe Geschwindigkeit haben.

Bei der Untersuchung von Noyes und Sammet waren die Bedingungen so eingestellt, dais die Entwickelung von Sauerstoff und die Regeneration der Säure nach Möglichkeit beschränkt waren. Bei Doumers Versuchen waren die Bedingungen so, dals die maximale Entwickelung von Sauerstoff stattfinden mufste und Doumers Schlufs, dafs $1 / 3$ des Stromes immer zur Elektrolyse der Chlorwasserstoffsäure benutzt wird, beruht hauptsächlich auf der Verallgemeinerung, dals das Verhältnis des anodischen Sauerstoffs zum kathodischen Wasserstoff konstant und unabhängig von der Stärke des Stromes und der Konzentration der Lösung ist.

In der zu beschreibenden Untersuchung haben wir weiter die Elektrolyse der Chlorwasserstoffsäure unter verschiedenen Bedingungen untersucht. Bei den in Tabelle 1 zusammengestellten Versuchen war der benutzte Apparat, der in Fig. 1 dargestellt ist, ein HoFFMans scher Apparat mit einem Hempenschen Niveaugefäls, so dafs die Gasmessungen bei atmosphärischem Druck ausgeführt werden konnten; die Elektroden waren durch die Gummistopfen eingeführt. Bei anderen Versuchen, die in Tabelle 1 unter B zusammengestellt sind, war der Apparat mit einer Anode versehen, die entweder aus einem Silberfiltertiegel mit einer Asbestschicht oder aus gefälltem Silber auf der Schicht eines Porzellanfiltertiegels bestand, wie in Fig. 2 gezeichnet ist. Dieser Apparat war so angeordnet, dafs Säure 
Tabelle 1.

Silberanoden.

\begin{tabular}{|c|c|c|c|c|c|c|c|}
\hline $\begin{array}{c}\text { Zeit } \\
\text { in } \\
\text { Minut. }\end{array}$ & $\begin{array}{l}\text { Strom } \\
\text { in } \\
\text { Amp. }\end{array}$ & \begin{tabular}{|c|} 
Span- \\
nung \\
in Volt
\end{tabular} & $\begin{array}{l}\text { Konz. in } \\
\text { Teilen } \\
\text { auf } 1000\end{array}$ & $\begin{array}{l}\text { Wasser- } \\
\text { stoff } \\
\text { in ccm }\end{array}$ & $\begin{array}{l}\text { Sauer- } \\
\text { stoff } \\
\text { in ccm }\end{array}$ & $\begin{array}{c}\text { Verhält- } \\
\text { nis von } \\
\mathrm{O}: \mathrm{H}\end{array}$ & $\begin{array}{l}\text { Angenähert. } \\
\text { Fläche der } \\
\text { Silberanode } \\
\text { in qeu }\end{array}$ \\
\hline
\end{tabular}

A

\begin{tabular}{l|l|l|l|l|l|l|l}
31 & 0.085 & $88-89$ & 0.73 & 23.94 & 3.44 & 0.143 & 1.0 \\
31 & 0.080 & $28-32$ & 0.73 & 22.40 & 2.60 & 0.116 & $21.4^{: 3}$ \\
30 & 0.080 & $83-90$ & 0.73 & 22.44 & 0.32 & 0.014 & $14.0^{4}$
\end{tabular}

B

\begin{tabular}{l|r|c|c|c|c|c|c}
30 & 0.050 & 90 & $0.36^{1}$ & 14.9 & 0.9 & 0.060 & $?$ \\
30 & $\left\{\begin{array}{l}0.050 \\
0.045\end{array}\right.$ & 95 & $0.36^{2}$ & 12.9 & 0.03 & 0.002 & $20^{3}$ \\
30 & 0.070 & $60-65$ & $0.73^{1}$ & 20.1 & 3.75 & $0.186^{3}$ & $20^{5}$ \\
30 & 0.070 & $62-65$ & $0.73^{2}$ & 20.2 & - & $0.000^{4}$ & $20^{6}$
\end{tabular}

von bekanntem Gehalt aus dem Niveaugefäfs eintreten konnte, um die Anodenflüssigkeit zu ersetzen, die langsam abfiltrierte, um die Zusammensetzung des Elektrolyten konstant zu halten, und um abgespültes und kolloides Silberchlorid zu sammeln.

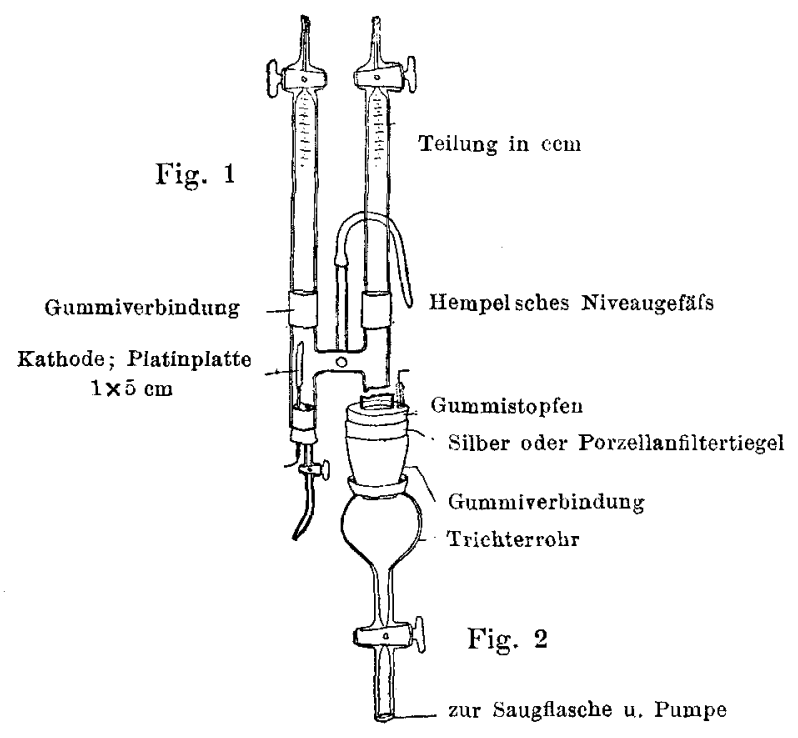

1 Fast N/50. - ${ }^{2}$ Fast N/100. - ${ }^{3}$ Anode vorher benutzt. - ${ }^{4}$ Frische Anodenfläche. - ${ }^{3}$ Ungefähr $3 \mathrm{~g}$ gefälltes Silber in Porzellan. - ${ }^{6}$ Silbertiegel. 
Aus den angeführten Resultaten ergibt sich, dals unter den gewählten Bedingungen sich Sauerstoff nur entwickelte, wenn die angreifbare Silberfiäche klein war, und dals seine Menge bei weitem nicht an die von Doumer angegebenen Beträge reichte. Obwohl DOUMER wenigstens hinsichtlich seiner früheren Versuche angibt, dafs kein Chlor und keine Sauerstoffsäure des Chlors sich in der Anodenflüssigkeit fanden, so war doch nach unserem Versuch, wenn Sauerstoff frei gemacht wurde, dessen Entwickelung immer wenigstens von erkennbaren Chlormengen begleitet. Silberoxyd im Gemisch mit Silberchlorid wurde auf der Anode bei jedem Versuch beobachtet und die Bildung des Oxyds begann sogleich mit dem Einsetzen der Elektrolyse. Es scheint klar, dafs solange die Anode eine grofse Silberfläche darbietet, die leicht angreifbar ist, sowohl Sauerstoff als Chlor sie angreifen. Das einmal festhaftende Silberchlorid bleibt auf der Anode, aber das Silberoxyd ist der Einwirkung der Chlorwasserstoffsäure und des freien Chlors im ionisierten oder molekularen Zustand ausgesetzt. Nur wenn die angreifbare Oberfläche hinreichend beschränkt wird, beginnt die Entwickelung des Sauerstoffs, und es wurde wiederholt bemerkt, dafs eine bereits vorhandene Sauerstoffentwickelung leicht unterbrochen werden konnte, wenn man die schützende Schicht auf der Anode zerstörte.

Die Bildung des leicht diffundierenden kolloidalen Silberchlorids, welche eintritt, wenn die freie Fläche der Silberanode beschränkt und das Potential zwischen den Elektroden ziemlich hoch ist, kann kaum auf die einfache Wirkung des Chlors auf Silber zurückgeführt werden, da sie nicht stattfindet, wenn eine Silberanodenfläche hinreichender Gröfse benutzt wird. Scheinbar geht die Bildung von Silberoxyd der Entstebung des kolloidalen Silberchlorids voraus. Wenn frisch gefälltes Silberchlorid der Einwirkung verdünnter Salzsäure unterworfen wird, so wird es nach unseren Versuchen krystallinischer und nicht kolloidal, während verdünnte Salzsäure auf Silberoxyd unter Bildung von fein verteiltem Silberchlorid wirkt, und die Bildung des wolkigen kolloidalen Silberchlorids tritt charakterischerweise ein, wenn Silberoxyd im Platindrahtnetz in Chlorwasser eingetaucht wird, wobei gleichzeitig lösliches Silberhypochlorit entsteht. Demnach scheint es, dals die Bedingungen, unter denen sich kolloidales Silberchlorid bei der Elektrolyse von Chlorwasserstoffsäure am wahrscheinlichsten bildet, dann auftreten, wenn Silberoxyd auf der Anode von Chlor angegriffen wird, wobei ein Teil des Silberchlorids durch direkte Einwirkung von Chlor auf Silberoxyd ent- 
steht und ein Teil durch die Wirkung von Chlorwasserstoffsäure auf lösliches Silberhypochlorit. Dies scheint uns wahrscheinlich die Reaktion zu sein, die in jedem Falle zur Bildung einer Wolke von kolloidalem Silberchlorid an der Anode Veranlassung gab bei den Uberführungsversuchen von NoxES und SAMMET $^{1}$ mit Lösungen der Chloride.

Bei den folgenden Versuchen war die Silberanode von einer Schicht von Silberchlorid bedeckt, um nach Möglichkeit die Fläche vor dem Angriff durch Sauerstoff zu schützen. Die Anode von Silberdraht, die $4 \mathrm{~cm}$ lang war und $2.27 \mathrm{~mm}$ im Durchmesser hatte, wurde zuerst in geschmolzenes Silberchlorid getaucht und dann als schnell rotierende Anode bei einer vorläufigen Elektrolyse von starker Chlorwasserstoffsäure benutzt, bis reichliche Chlorentwickelung stattfand.

Auf diese Weise wurde die Anode unangreifbar gemacht, abgesehen von einigen unvollständig bedeckten Punkten mit dem praktischen Effekt, dafs die aktive Fläche sehr beschränkt wurde und beiläufig die Stromdichte für gegebene Stromstärken vergröfsert wurde. Nicht jede auf diese Weise hergestellte Anode war vollkommen genug, um bei einem Versuch ohne Bildung von kolloidalem Silberchlorid dienen zu können, aber einige bruuchbare Anoden wurden so erhalten und mit diesen wurden die angeführten Versuche ausgeführt. Für diese Versuche wurde der Apparat so verändert, dals er die Bewegung der Elektroden und eine entsprechende Regulierung des Potentials zwischen den Elektroden für verschiedene Konzentrationen ermöglichte. Der Apparat ist in Fig. 3 gezeichnet. Die Ergebnisse sind in Tabelle 2 zusammengestellt.

Bei diesen Versuchen wurde das Verbältnis von Sauerstoff zu Wasserstoff innerhalb der bestimmten Grenzen zwar nicht wesentlich oder regelmä̊sig durch Änderungen der Stromstärke oder des Potentials zwischen den Elektroden oder der Stromdichte geändert. aber es schien merklich zu wachsen, wenn die Konzentration der Lösung abnahm. Nur bei den niedrigsten Konzentrationen von 0.43 Teilen in 1000 und mit einer Anode von sehr beschränktem aktiven Querschnitt war der mittlere Wert so hoch wie der von DOOMER erhaltene. Unsere Resultate weichen demuach in dieser Hinsicht von denen Doumers ab. Wir finden, dafs das Verhültnis der Volumina von Sauerstoff und Wasserstoff bei der Elektrolyse von

1. e. 
Tabelle 2.

Anoden von Silber, geschützt durch Silberchlorid.

\begin{tabular}{c|c|c|c|c|c|c}
\hline \hline $\begin{array}{c}\text { Zeit } \\
\text { in } \\
\text { Min. }\end{array}$ & $\begin{array}{c}\text { Strom } \\
\text { in } \\
\text { Amp. }\end{array}$ & $\begin{array}{c}\text { Span- } \\
\text { nung } \\
\text { in Volt }\end{array}$ & $\begin{array}{c}\text { Konz. } \\
\text { Teile } \\
\text { in } 1000\end{array}$ & $\begin{array}{c}\text { Wasserstoff } \\
\text { in ccm }\end{array}$ & $\begin{array}{c}\text { Sauer- } \\
\text { stoff } \\
\text { in ccm }\end{array}$ & $\begin{array}{c}\text { Verhältuis } \\
\text { O : H }\end{array}$ \\
\hline \hline 30 & 0.05 & 11.3 & 4.3 & 14.9 & 3.6 & 0.242 \\
30 & 0.05 & 12.5 & 0.86 & 13.6 & 4.0 & 0.294 \\
30 & 0.05 & 12.5 & 0.43 & 14.8 & 5.0 & 0.333 \\
30 & 0.10 & 21.0 & 0.86 & 26.1 & 6.4 & 0.245 \\
30 & 0.10 & 21.6 & 0.86 & 25.5 & 6.7 & 0.263 \\
30 & 0.10 & 21.3 & 086 & 24.6 & 8.0 & 0.325 \\
30 & 0.10 & 20.3 & 0.43 & 26.3 & 8.3 & 0.316 \\
30 & 0.10 & 21.6 & 0.43 & 25.0 & 8.3 & 0.332 \\
30 & 0.15 & 26.6 & 4.3 & 39.7 & 10.5 & 0.264 \\
30 & 0.15 & 28.5 & 0.86 & 37.5 & 11.8 & 0.315 \\
30 & 0.15 & 29.5 & 0.43 & 379 & 12.5 & 0.330 \\
\end{tabular}

Chlorwasserstoffsäure direkt von der Konzentration der Lösung abhängt, und weiterhin, dafs bei einer Konzentration von 0.43 Teilen

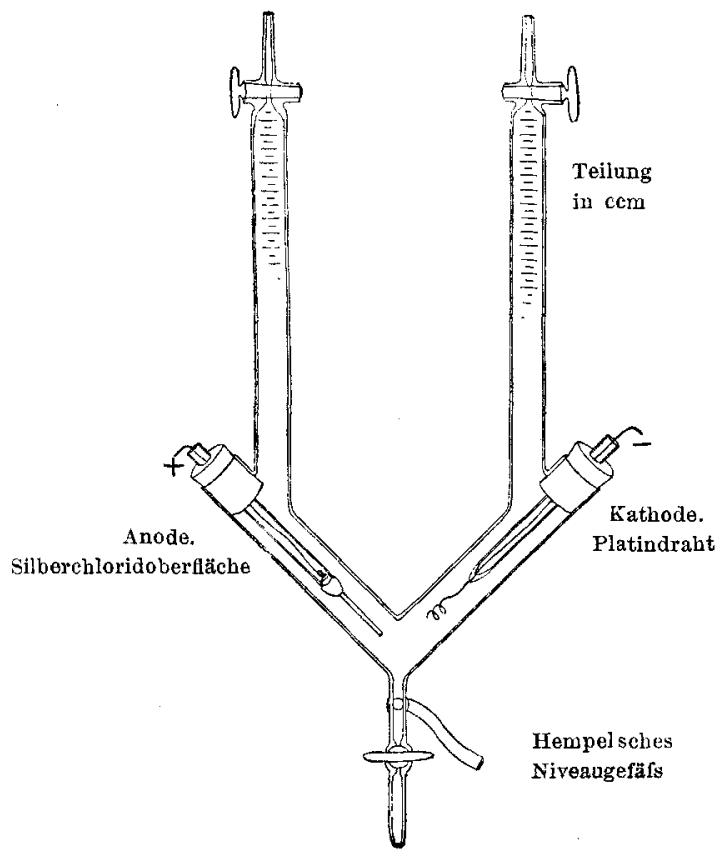

Fig. 3.

Z. anorg. Chom. Bd. 64 . 
in 1000 Dơmers Verhältnis nur dann erhalten wird, wenn die Anode weitgehend von der Einwirkung von Chlor und Sauerstoff geschützt wird.

Wenn dies der Fall ist, so ist es von Interesse, herauszufinden, wie diese mit der geschützten Silberanode erhaltenen Resultate sich zu denen stellen, die man mit Platinanoden für ähnliche Konzentrationen der Lösungen erhält. In der folgenden Tabelle sind die Einzelheiten der Versuche mit einer Platindrahtspirale $(0.8 \mathrm{~mm} \times 5 \mathrm{~cm})$ als Anode und Lösungen ähnlicher Konzentrationen mitgeteilt.

Tabelle 3.

Platinanode.

\begin{tabular}{c|c|c|c|c|c|c|c|c}
\hline \hline $\begin{array}{c}\text { Zeit } \\
\text { in }\end{array}$ & $\begin{array}{c}\text { Strom } \\
\text { in }\end{array}$ & $\begin{array}{c}\text { N.D } \\
\text { in }\end{array}$ & $\begin{array}{c}\text { Span- } \\
\text { nung }\end{array}$ & $\begin{array}{c}\text { Konz. } \\
\text { Teile }\end{array}$ & $\begin{array}{c}\text { Wasser- } \\
\text { stoff }\end{array}$ & $\begin{array}{c}\text { Sauer } \\
\text { stoff }\end{array}$ & $\begin{array}{c}\text { Ver- } \\
\text { hältnis }\end{array}$ & $\begin{array}{c}\text { Mittel für } \\
\text { jede }\end{array}$ \\
Minut. & Amp. & Amp. & in Volt & in 1000 & in ecm & in cem & v. H:O & Konzentr. \\
\hline
\end{tabular}

Anodenfläche $1.25 \mathrm{qcm}$

\begin{tabular}{l|r|r|r|r|r|r|l|c}
30 & 0.10 & 8.0 & 4.0 & 43.0 & 27.6 & 2.3 & 0.083 & 0.083 \\
30 & 0.05 & 4.0 & 12.0 & 4.3 & 14.1 & 2.3 & 1.63 & - \\
30 & 0.10 & 8.0 & 20.5 & 4.3 & 26.2 & 3.7 & 1.41 & - \\
30 & 0.15 & 12.0 & 28.2 & 4.3 & 38.8 & 6.2 & 1.59 & 0.154 \\
30 & 0.05 & 4.0 & 13.2 & 0.86 & 15.2 & 4.4 & 0.289 & - \\
30 & 0.10 & 80 & 21.2 & 0.86 & 28.6 & 7.1 & 0.242 & - \\
30 & 0.15 & 12.0 & 30.0 & 0.86 & 39.4 & 10.6 & 0.268 & 0.266 \\
30 & 0.05 & 4.0 & 14.0 & 0.43 & 14.4 & 4.8 & 0.333 & - \\
30 & 0.10 & 8.0 & 20.8 & 0.43 & 25.6 & 7.9 & 0.308 & - \\
30 & 0.15 & 12.0 & 28.3 & 0.43 & 37 & 11.9 & 0.321 & 0.320
\end{tabular}

Es ist offenbar, dafs für die niedrigen Konzentrationen 0.86 Teile und 0.43 Teile in 1000 die Verhältnisse der Volumina von Sauerstoff und Wasserstoff im Mittel nur wenig niedriger sind als die mit einer geschützten Silberanode erhaltenen. Für die höheren Konzentrationen, 4.3 Teile in 1000 , ist das Verhältnis viel kleiner als bei einer Silberanode, wahrscheinlich deswegen, weil bei der höheren Konzentration der Säure der Einflufs der sehr viel höheren aber unbestimmbaren Stromdichte auf der Silberanode von Wichtigkeit sein kann. Die Verhältnisse, die mit der Platinanode erhalten wurden, sind niemals höher als die mit der Silberanode und diese T'atsache führte zur Anwendung der Platinanode in Lösungen noch geringerer Konzentration, denn obwohl bei den beschriebenen Versuchen die geschützte Silberanode praktisch un- 
angreifbar war bei den zur Elektrolyse zur Lösungen von der benutzten Konzentration erforderlichen Stromdichten, so zeigte sich doch, dafs die Chloridschicht der Anode zerstört wurde, als man die Versuche auf die Elektrolyse von Chlorwasserstofflösungen viel geringerer Konzentration ausdehnte. Bei der Untersuchung von Lösungen aufserordentlicher Verdünnung wurde es demnach notwendig, zu Platinanoden zurückzukehren, was sich auch als ganz zweckmälsig erwies. Tabelle 4 gibt die Einzelheiten eines Versuches mit einer Lösung, die 0.043 Teile in 1000 enthielt; es kam eine Spiralanode und Kathode von Platindraht mit $0.8 \mathrm{~mm}$ Durchmesser und $5 \mathrm{~cm}$ Länge in dem Apparat der Fig. 3 zur Anwendung. Das an der Anode während der ersten 30 Minuten (A) entwickelte Gas wurde gemessen in Zwischenräumen von 5 Minuten und als Sauerstoff betrachtet ohne Korrektur für möglicherweise beigemischtes Chlor. Der Versuch wurde in ähnlicher Weise 45 Minuten fortgesetzt (B) und nach dieser Zeit wurde das Anodengas entfernt, mit Natriumbydroxyd gewaschen und in einer Hexpesu schen Bürette gemessen. Die Volumenverminderung betrug $0.5 \mathrm{~cm}$ auf die ursprünglich gefundenen 25.4. Am Ende einer weiteren Periode (C) von 45 Minuten wurde das Anodengas wieder abgezogen und erwies sich bei der Prüfung mit Kaliumjodid als frei von Chlor. So ist es wahrscheinlich, dafs die geringe Verminderung, die man beim Waschen des Anodengases der beiden ersten Zeiträume mit Natriumbydroxyd fand, eher der Löslichkeit des Sauerstoffs als der Gegenwart von Chlor zuzuschreiben war. Die Einzelheiten der Ablesungen nach je 5 Minuten und am Ende sind in der Tabelle 4 zusammengestellt.

Bei diesem Versuch war das Verhältnis von Sauerstoff zu Wasserstoff während der ganzen Elektrolyse ziemlich konstant und ungefähr $30 \%$ höher als Doumers Verhältnis und als das Maximum, das man bei den niedrigsten früher angewandten Konzentrationen der Lösungen erhielt. Es ist überdies von Interesse, festzustellen, dals die $91.2 \mathrm{ccm}$ Wasserstoff, die sich in 2 Stunden entwickelten, das Äquivalent von ungefähr $0.276 \mathrm{~g}$ Chlorwasserstoffsäure sind, d. h. von ungefähr $20 \times 0.014 \mathrm{~g}$, die ursprünglich in $330 \mathrm{ccm}$ der Lösung enthalten waren. Wenn aller Wasserstoff aus der primären Elektrolyse von Chlorwasserstoff stammte, so muls von der letzteren 20 mal so viel als ursprünglich vorhanden war, elektrolysiert und im Laufe der Versuche regeneriert sein.

Die Einzelheiten ähnlicher Versuche mit Platinelektroden von 


\section{Tabelle 4.}

Platinanode: Fläche $1.25 \mathrm{qcm}$. Volumen der Lösung $=330 \mathrm{ccm}$.

\begin{tabular}{c|c|c|c|c|c|c|c}
\hline \hline $\begin{array}{c}\text { Zeit } \\
\text { in }\end{array}$ & $\begin{array}{c}\text { Strom } \\
\text { in }\end{array}$ & $\begin{array}{c}\text { N.D.100 } \\
\text { in }\end{array}$ & $\begin{array}{c}\text { Span- } \\
\text { nung }\end{array}$ & $\begin{array}{c}\text { Konz. } \\
\text { Teile } \\
\text { Minut. }\end{array}$ & $\begin{array}{c}\text { Wasser- } \\
\text { stoff } \\
\text { Amp. }\end{array}$ & $\begin{array}{c}\text { Sauer- } \\
\text { Amp. } \\
\text { in Volt }\end{array}$ & $\begin{array}{c}\text { Ver- } \\
\text { hältmis } \\
\text { in } 1000\end{array}$ \\
\hline
\end{tabular}

A

\begin{tabular}{c|l|l|l|} 
Beginn & 0.07 & 5.6 & 87 \\
5 & 0.095 & 7.6 & 87.5 \\
10 & 0.105 & 8.4 & 87 \\
15 & 0.105 & 8.4 & 87 \\
20 & 0.105 & 8.4 & 87 \\
25 & 0.100 & 8.0 & 86.5 \\
30 & 0.100 & 8.0 & 86.5
\end{tabular}

\begin{tabular}{|c|c|c|c}
0.043 & - & - & - \\
0.043 & 3.7 & 1.7 & - \\
0.043 & 4.0 & 1.7 & - \\
0.043 & 3.9 & 1.8 & - \\
0.043 & 4.1 & 1.7 & - \\
0.043 & 4.0 & 1.7 & - \\
0.043 & 4.1 & 1.7 & - \\
\hline & 23.8 & $10.3^{1}$ & 0.433
\end{tabular}

B

\begin{tabular}{r|l|l|l}
5 & 0.100 & 8.0 & 85 \\
10 & 0.100 & 8.0 & 85 \\
15 & 0.100 & 8.0 & 8 \\
20 & 0.095 & 7.6 & 8 \\
25 & 0.105 & 8.4 & 8 \\
30 & 0.100 & 8.0 & 8 \\
35 & 0.095 & 7.6 & 8 \\
40 & 0.100 & 8.0 & 85 \\
45 & 0.100 & 8.0 & 85
\end{tabular}

\begin{tabular}{l|l}
85 & 0.0 \\
85 & 0.0 \\
84 & 0.0 \\
86 & 0.0 \\
87 & 0.0 \\
86 & 0.0 \\
86 & 0.0 \\
85 & 0.0 \\
85 & 0.0
\end{tabular}

\begin{tabular}{l|l|l|l}
0.043 & 3.9 & 1.7 & - \\
0.043 & 4.0 & 1.6 & - \\
0.043 & 3.7 & 1.6 & - \\
0.043 & 4.1 & 1.8 & - \\
0.043 & 4.0 & 1.7 & - \\
0.043 & 3.7 & 1.7 & - \\
0.043 & 4.0 & 1.8 & - \\
0.043 & 3.5 & 1.6 & - \\
0.043 & 3.7 & 1.6 & - \\
\hline & 34.6 & $15.1^{2}$ & 0.436
\end{tabular}

\begin{tabular}{r|l|}
5 & 0.09 \\
10 & 0.09 \\
15 & 0.09 \\
20 & 0.09 \\
25 & 0.09 \\
30 & 0.09 \\
35 & 0.09 \\
40 & 0.085 \\
45 & 0.100
\end{tabular}

\begin{tabular}{l|l|}
7.2 & 90 \\
7.2 & 87 \\
7.2 & 90 \\
7.2 & 90 \\
7.2 & 89 \\
7.2 & 87.5 \\
72 & 87 \\
6.8 & 87 \\
8.0 & 87
\end{tabular}

$\mathrm{C}$

\section{\begin{tabular}{l|l}
0.043 & 3.4
\end{tabular}} $0.043 \quad 3.6$ \begin{tabular}{l|l}
0.043 & 3.7
\end{tabular} \begin{tabular}{l|l}
0.043 & 3.6
\end{tabular} \begin{tabular}{l|l}
0.043 & 3.7
\end{tabular} $0.043 \quad 3.6$ 0.043 0.043 0.043

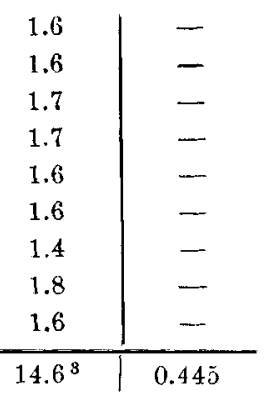

verschiedenem Querschnitt und in Lösungen von noch geringerer Konzentration sind in der folgenden Tabelle zusammengestellt.

${ }^{1}$ Nicht korr. für Chlor.

Nicht korr. für Chlor. Nach Waschen mit $\mathrm{NaOH}$ wurden die $25.4 \mathrm{ccm}$ von $A$ und $B$ auf $24.9 \mathrm{~cm}$ reduziert.

${ }^{3}$ Erwies sich durch $\mathrm{KJ}$ als frei von Chlor. 
Unter A stehen die Ergebnisse bei verschiedenen Konzentrationen mit einer Anode von kleinem Querschnitt, während unter B diejenigen mit einer Anodenfläche, die $80 \mathrm{mal}$ so grofs war, zusammengestellt sind.

Tabelle 5 .

Platinanode.

\begin{tabular}{c|c|c|c|c|c|c|c|c|}
\hline \hline Zeit & Strom & N.D. roo & Span- & Konz. & Wasser- & Sauer- & Verhältnis & Mittel \\
in & in & in & nung & Teile & stoff & stoff & von & für jede \\
Min. & Amp. & Amp. & in Volt & in 1000 & in ecm & in ccm & $\mathrm{O}: \mathrm{H}$ & Konz. \\
\hline
\end{tabular}

A

\begin{tabular}{l|l|l|r|r|r|r|r|c}
\multicolumn{10}{c}{ Anodenfläche $=1.25$ qcm. } \\
30 & 0.05 & 4.0 & 23.4 & 0.172 & 14.6 & 5.7 & 0.390 & - \\
30 & 0.10 & 8.0 & 85.7 & 0.172 & 24.2 & 9.9 & 0.409 & 0.399 \\
30 & 0.05 & 4.0 & 97.2 & 0.086 & 11.0 & 5.0 & 0.454 & - \\
30 & 0.10 & 8.0 & 87.3 & 0.086 & 24.7 & 11.1 & 0.468 & 0.461 \\
30 & 0.05 & 4.0 & 95.2 & 0.043 & 12.2 & 5.4 & 0.442 & 0.442 \\
30 & 0.039 & 3.12 & 98.7 & 0.0086 & 8.6 & 4.1 & 0.477 & 0.477
\end{tabular}

B

\begin{tabular}{l|l|l|l|l|l|l|l|l}
30 & 0.10 & 0.10 & 86.7 & 0.172 & 24.0 & 9.1 & 0.379 & - \\
30 & 0.05 & 0.05 & 95.8 & 0.086 & 12.8 & 5.2 & 0.406 & - \\
30 & 0.05 & 0.05 & 92.8 & 0.093 & 11.6 & 4.7 & 0.405 & - \\
30 & 0.031 & 0.031 & 95.8 & 0.0086 & 8.8 & 3.7 & 0.420 & -
\end{tabular}

Die Versuche jeder dieser Reihen bestätigen im allgemeinen den früheren Beweis dafür, dals das Verhältnis des freigemachten Sauerstoffs zunimmt, wenn die Konzentration der Lösung abnimmt. Das höchste Verhältnis von Sauerstoff zu Wasserstoff, das man bei der niedrigsten Konzentration, 0.0086 Teile in 1000, und bei der höchsten Stromintensität auffand, fällt bis auf $5 \%$ mit dem Verhältnis zusammen, das man finden würde, wenn Wasser der primäre und einzige Elektrolyt wäre. Ein Vergleich der beiden Reihen zeigt, dafs bei sehr starker Zunahme der Fläche der Anode die Menge des freigemachten Sauerstoffs etwas abnimmt, oder im allgemeinen eine 80 fache Zunahme der Stromdichte bedingt im Mittel eine $10 \%$ ige Zunahme des Sauerstoffverhältnisses.

Man erkennt, dafs die experimentellen Ergebnisse, von denen hier berichtet wurde, DonMers Anspruch widersprechen, dafs das Verbältnis der bei der Elektrolyse von Chlorwasserstoffsäure entwickelten Volumina von Wasserstoff und Sauerstoff konstant und 
unabhängig von Stromstärke und Konzentration der Lösung seien. Sie liefern demnach keine Basis für Docmers Berechnung der gleichen Geschwindigkeit für das Wasserstoff- und Chlorion. Auch Doumers Entdeckung der gleichen Acidität an den Elektroden führt nicht zu einer solchen Beziehung der Geschwindigkeiten, denn wenn man annimmt, dafs der Strom durch die Ionen der Salzsäure transportiert wird, so haben wir 166.6 nach Nores und SAMmET ${ }^{1}$ für die Überführungszahl des Chlors bei einer Konzentration, wo sich das Sauerstoff-Wasserstoffverbältnis Doumers Zahl 0.332 nähert; und wenn man weiter annimmt, dafs der entwickelte Sauerstoff frei gemacht wird durch die Einwirkung der überführten Chlorionen unter gleichzeitiger Regeneration der Salzsäure, so muls die Bedingung, die die Entwickelung des Sauerstoffs entsprechend dem Sauerstoff-Wasserstoffverbältnis von 0.332 hervorruft, auch die gleiche Acidität der Elektrodenflüssigkeiten bedingen.

Die beobachteten Erscheinungen liefern demnach kein Kriterium zur Entscheidung darüber, wie viel von dem frei gemachten Sauerstoff bei der Elektrolyse von Chlorwasserstoffsäure unter gegebenen Bedingungen durch die Flüssigkeit übergeführt wird, und wie viel durch die Wirkung des übergeführten Chlors an der Anode frei gemacht wird.

1. c.

New Haven, U. S. A., The Kent Chemical Laboratory of Yale Dnicersity.

Bei der Redaktion eingegangen am 4. August 1909. 